\title{
Impact of COVID-19 lockdown on glycemic control in children and adolescents
}

\author{
Abdulmoein E. Al Agha, FRCPCH, Razan S. Alharbi, MBBCH, Omar A. Almohammadi, MBBCH, \\ Sondos Y. Yousef, MBBCH, Ahad E. Sulimani, MBBCH, Rawan A. Alaama, MBBCH.
}

\begin{abstract}
الأهداف : دراسة مدى تأثير جائحة كورونا على الأطفال واليافعين المصابين بداء

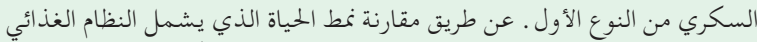

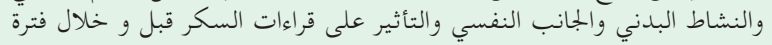
الحجر المنزليز

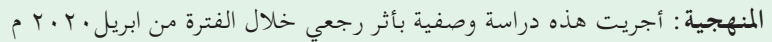

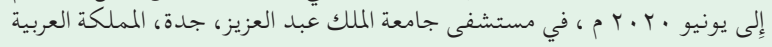

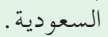

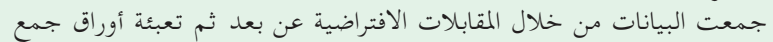

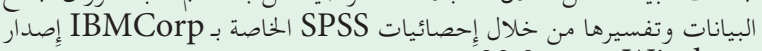
20.0 النسخة Windows

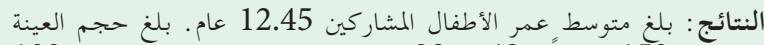

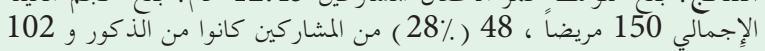

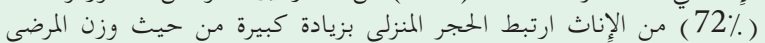

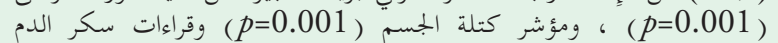
( )

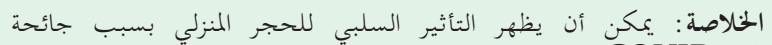

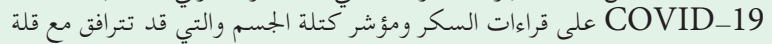

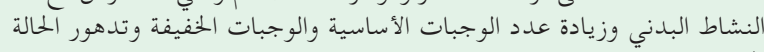
المزاجية.
\end{abstract}

Objectives: To identify how children and adolescents with type 1 diabetes were coping with their condition during the COVID-19 lockdown, by detecting differences in blood glucose control and in lifestyle, including diet, physical activity, and mood deterioration, before and during the lockdown.

Methods: This descriptive, cross-sectional study was conducted between April and June 2020 at King Abdulaziz University Hospital (KAUH), Jeddah, Saudi Arabia. Data were collected from interviews, using various forms of telecommunication.

Results: The total sample size was 150 patients, 48 (28\%) of whom were males and $102(72 \%)$ females. The mean age of the patients was 12.45 years. The lockdown was associated with a significant increase in patients' weight $(p=0.001)$, body mass index $(p=0.001)$, and blood glucose readings $(p=0.007)$ compared to their values before the lockdown.
Conclusion: A negative impact of the COVID-19 lockdown was found on blood glucose values and BMI, which may correlate with a lack of physical activity, increased consumption of carbohydrates and fast food, and mood deterioration.

Keywords: type 1 diabetes, COVID-19, glycemic control

Saudi Med J 2021; Vol. 42 (1): $44-48$

doi: 10.15537/smj.2021.1.25620

From the Pediatric Department (Al Agha), Faculty of Medicine (Alaama), King Abdulaziz University; and from the Faculty of Medicine (Alharbi, Almohammadi, Yousef, Sulimani) Ibnsina National College, Jeddah, Kingdom of Saudi Arabia.

Received 24th September 2020. Accepted 7th December 2020.

Address correspondence and reprint request to: Dr. Abdulmoein Al Agha, Professor, Pediatric Department, Faculty of Medicine, King Abdulaziz University, Jeddah, Kingdom of Saudi Arabia. E-mail: aagha@kau.edu.sa

$T$ he most frequent chronic metabolic disorder in children is diabetes mellitus. Hyperglycemia is the main characteristic of diabetes mellitus, which is attributed to insulin deficiency due to pancreatic $\beta$-cell loss. ${ }^{1,2}$ It is a lifelong illness that requires medical therapy, multiple daily administrations of insulin, a healthy diet, exercise, and psychological support. ${ }^{3,4}$ The control of type 1 diabetes (T1D) may be affected by many factors, and it is important to reduce the risk of complications and infections. ${ }^{5}$ It was postulated that, during the stressful time of the coronavirus (COVID-19) pandemic, managing diabetes could be difficult, especially for children with T1D., In addition, the pandemic induced some challenging lifestyle changes, including dietary habits, physical activity, insulin dose adjustment, and access to insulin supply. All of these changes could adversely affect the control of their disease. Recently, new approaches are being studied to provide new treatments to diabetic patients. Some studies have been conducted to explore 
how diabetic patients are dealing with their diabetes during the COVID-19 lockdown; ${ }^{7-9}$ however, few have been conducted in Saudi Arabia. We aimed to assess how children and adolescents with T1D were coping with their diabetes during the lockdown, and more broadly, to offer the diabetic community better strategies for healthy living in lockdown.

Methods. This was a descriptive, cross-sectional study on children and adolescents with T1D, conducted at King Abdulaziz University Hospital (KAUH) in Jeddah, Saudi Arabia, between April and June 2020. Subjects were contacted via an online virtual pediatric endocrine outpatient clinic at KAUH and interviewed. The study was approved by the KAUH Ethical Committee. The patients' data were kept safe in the secure office of the principal investigator, ensuring the protection and anonymity of patients' identities and accessed only by the authors. Informed consent was obtained from the patients' parents prior to their participation in the study.

The interview was designed to identify and assess the following: i) demographic characteristics and anthropometric measurements, including weight and height, comparing the data from their clinic visits within the last 3 months before lockdown and the first clinical visit during the lockdown. ii) The status of the patient's blood glucose control before and during the lockdown. This was achieved by asking about daily home glucose readings, and frequency of home glucose monitoring, assessed 3 months before lockdown and 3 months into lockdown. iii) Glycosylated hemoglobin A1c (HbA1c) level, measured before lockdown and at the end of lockdown (lockdown lasted 3 months, which was long enough to evaluate the results) and DKA (diabetic ketoacidosis) episodes. iv) Lifestyle, including dietary habits. Data were obtained by asking about the number of basic meals and snacks each patient had, the type of diet, and any difficulty encountered in following a healthy diet. Multiple questions were asked of the adolescents themselves or their parents regarding the patients' physical activity, including the baseline number of minutes of physical activity before lockdown, how it was affected during the lockdown, and satisfaction with its level. v) The insulin therapy regimen, types and frequency of injections before and during lockdown,

Disclosure. Authors have no conflict of interests, and the work was not supported or funded by any drug company. insulin dose adjustment, and difficulties in accessing insulin supply.

All children and adolescents with T1D aged 2-18 years, of both genders and all ethnicity, were included. Patients with type 2 diabetes, cognitive disability, language barriers, chronic complications, and comorbidities were excluded.

Statistical analysis. Data entry and analysis were performed using the Statistical Package for Social Sciences, version 23 (IBM Corp, Armonk, NY, USA). Data were compared before and during the lockdown. Quantitative data were expressed as a mean and standard deviation $( \pm S D)$, and simple descriptive statistics were applied to report qualitative variables as frequencies and percentages. A paired t-test was utilized to check the presence of significant differences before and during the lockdown. Results having a p-value of less than 0.05 were considered to be statistically significant.

Results. Baseline demographic and clinical characteristics. This study enrolled 150 patients, with a mean age of $12.45 \pm 4.62$ years. Forty-eight $(28 \%)$ of the patients were males, and $102(72 \%)$ were females. The mean weight was $40.73 \pm 15.49 \mathrm{~kg}$, and the mean body mass index (BMI) was $20.36 \pm 4.53 \mathrm{~kg} / \mathrm{m}^{2}$. The mean age at diagnosis with T1D was $8.23 \pm 5.34$ years. The mean glucose reading before the lockdown was $182.29 \pm 76.68 \mathrm{mg} / \mathrm{d}$, and the mean reading of HbAlc was $7.45 \pm 1.67 \%$. Table 1 presents the demographic and clinical profile of the patients. Approximately $10.7 \%$ of the patients did not analyze their glucose daily, $21.3 \%$ of patients analyzed their glucose 2-3 times daily, $14 \%$ analyzed their glucose $4-5$ times daily, $14.7 \%$ analyzed their glucose 5-7 times daily, and 39.3\% had a continuous glucose sensor (Figure 1). During the lockdown, the frequency of glucose monitoring has been changed in $48.8 \%$; of them $75.6 \%$ reported an increased frequency of glucose monitoring, and $24.4 \%$ reported a reduced frequency. Interestingly, our findings demonstrated that the percentages of patients who experienced DKA were decreased from $34.5 \%$ pre the lockdown to $10.7 \%$ during the lockdown. Of the study population, $15.3 \%$ took insulin twice a day, $50.7 \%$ took insulin 3 times a day, and $24.7 \%$ took insulin more than 4 times a day before the lockdown compared to $32.7 \%$ during the lockdown. In addition, $9.3 \%$ took insulin administered by an insulin pump.

Change from baseline due to the lockdown. Table 2 illustrates changes in weight and diabetic parameters from the baseline due to lockdown. 
COVID-19 lockdown and glycemic control ... Al Agha et al

Table 1 - Demographic data and anthropometric measurement (n=150).

\begin{tabular}{lc}
\hline Parameters & Mean \pm SD \\
\hline Gender (\%) & \\
$\quad$ Male & $48(28)$ \\
Female & $102(72)$ \\
Age (years) & $12.45 \pm 4.62$ \\
Height (centimeters) & $138.96 \pm 20.59$ \\
Weight, kg & $40.73 \pm 15.49$ \\
BMI, kg/m ${ }^{2}$ & $20.36 \pm 4.53$ \\
Age of diagnosis (years) & $8.09 \pm 4.89$ \\
\hline
\end{tabular}

Table 2 - A comparison of weights and diabetic parameters before and during lockdown.

\begin{tabular}{lcc}
\hline Variable & Mean \pm SD & $P$-value \\
\hline Weight $(\mathrm{kg})$ & & $<0.001^{*}$ \\
$\quad$ Before lockdown & $40.73 \pm 15.49$ & \\
$\quad$ During lockdown & $42.09 \pm 15.65$ & \\
BMI $(\mathrm{kg} / \mathrm{m})$ & & $<0.001^{*}$ \\
$\quad$ Before lockdown & $20.36 \pm 4.53$ & \\
$\quad$ During lockdown & $21.12 \pm 4.90$ & \\
Glucose reading $(m g / d L)$ & & $0.007^{*}$ \\
$\quad$ Before lockdown & $182.09 \pm 76.68$ & \\
$\quad$ During lockdown & $200.45 \pm 79.97$ & \\
HbAlc $(\%)$ & & \\
$\quad$ Before lockdown & $7.45 \pm 1.67$ & \\
During lockdown & $7.40 \pm 1.54$ & \\
\hline & *Significant at level 0.05 &
\end{tabular}

*Significant at level 0.05
Table 3 - Dietary patterns of the patients before and during the lockdown

\begin{tabular}{lcc}
\hline Variables & n & $(\%)$ \\
\hline Following healthy diet before lockdown & 72 & $(52.2)$ \\
Following healthy diet during the lockdown & 33 & $(34.9)$ \\
Healthy diet affected by lockdown & 32 & $(23.2)$ \\
Difficulty following diet during lockdown & 64 & $(46.4)$ \\
\hline
\end{tabular}

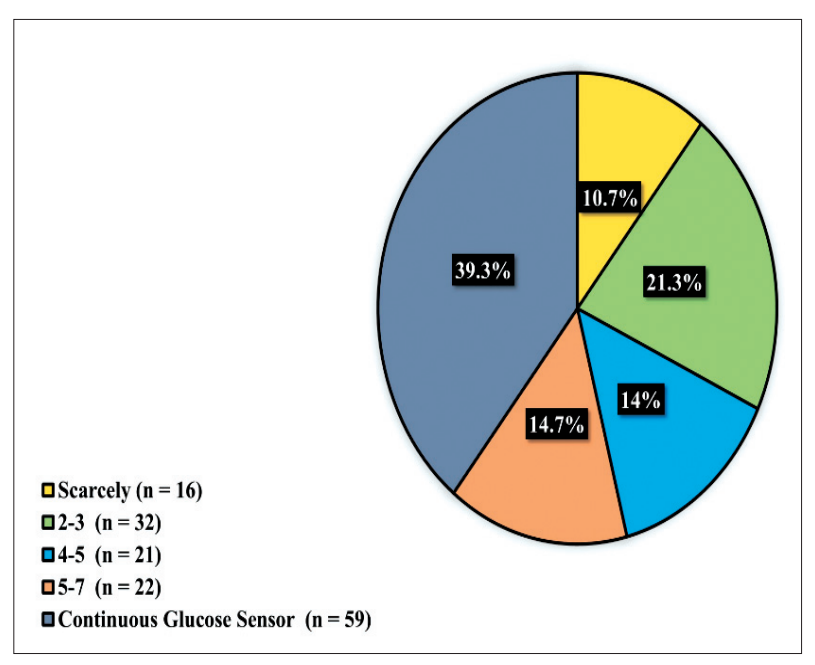

Figure 1 - Daily frequency of home glucose analysis.

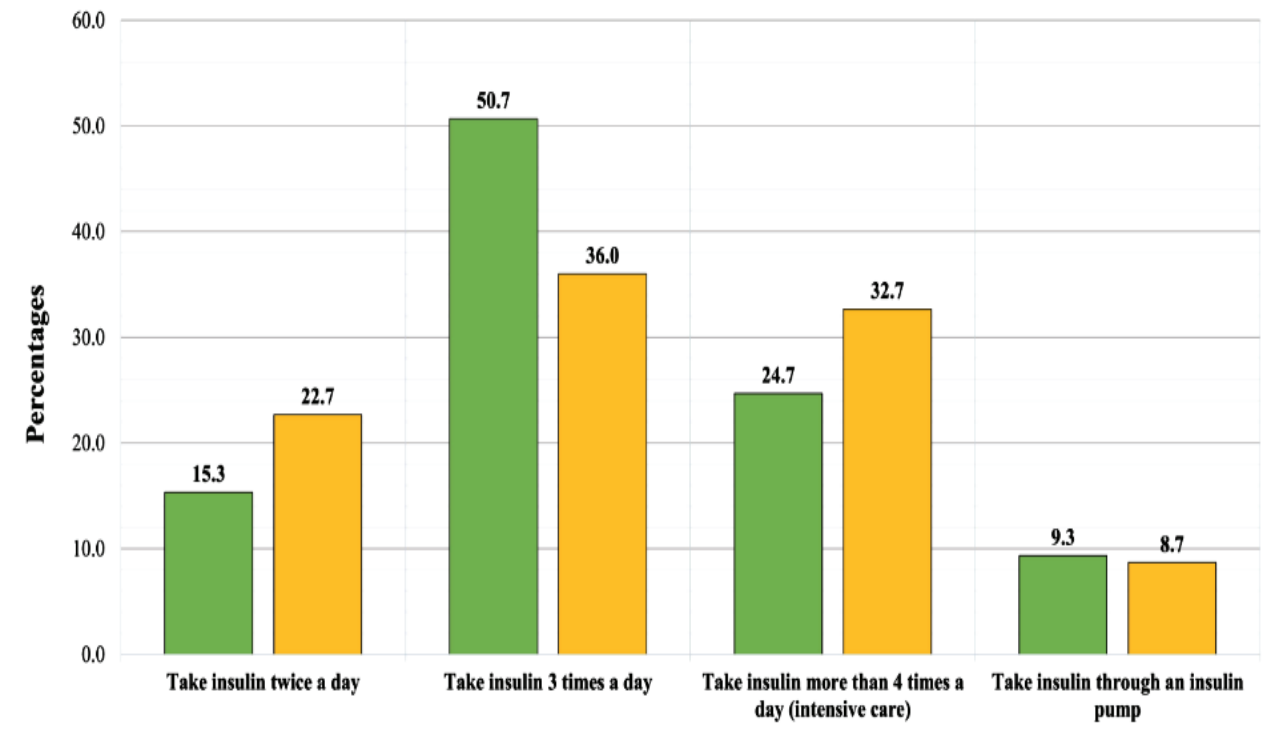

Figure 2 - Comparison between the number of insulin taking before and during the lockdown. 
Table 4 - Physical activity of patients before and during lockdown.

\begin{tabular}{lc}
\hline Variables & Percentage \\
\hline Duration of physical activity before the lockdown & \\
$\quad<30$ minutes & 40.5 \\
30-60 minutes & 28.0 \\
$\quad$ Not practicing & 27.4 \\
Physical activity affected during the lockdown & \\
$\quad$ Decreased & 66.1 \\
$\quad$ Increased & 19.0 \\
$\quad$ Not affected & 14.9 \\
Satisfaction with physical activity during lockdown & 48.0 \\
(n=34) & \\
\hline
\end{tabular}

Figure 2 shows the comparison between the number of insulin taking before and during the lockdown.

Regarding diet, Table 3 shows that $23.2 \%$ of the subjects were following a healthy diet before the lockdown, whereas $46.4 \%$ found difficulty in following a healthy diet during the lockdown for various reasons, such as a larger number of meals (47.4\%) and increased consumption of carbohydrates (37.1\%), fast food $(15.5 \%)$, and soft drinks $(9.3 \%)$. More than half $(52.2 \%)$ of the subjects reported that their diet had been affected by the lockdown. However, most of the patients were consuming the same number of snacks per day before and during the lockdown.

Table 4 shows the difference between the physical activity pre and during the lockdown, physical activity was also affected. During the lockdown, we noticed a decrease in their daily activity, increased their daily physical activity, and reported no change in their physical activity. And it was found that $64.9 \%$ were not satisfied with their level of physical activity.

Discussion. Since the lockdown began in March $2020,{ }^{10}$ several studies have been published about its effect on the lifestyle of children and adolescents. ${ }^{11}$ These data showed significant changes in their lifestyle habits. ${ }^{12}$ It is believed that the pandemic may have a lasting impact on physical activity patterns and dietary habits ${ }^{13}$ and therefore on the overall health of children/adolescents, especially for T1D patients and their glycemic control. Various factors have been shown to have a beneficial effect on T1D patients by improving their glycemic control, such as insulin therapy, glucose monitoring, regular exercise, following the recommendations about diet, and maintaining a healthy weight. The present study aimed to analyze the impact of the lockdown on these factors and explore how lifestyle changes reflected on the subjects' glycemic control.

Maintaining a healthy weight has obvious health benefits in improving glycemic outcomes. However, maintaining a healthy weight during the lockdown has become difficult, particularly for T1D children. According to studies, approximately half of the patients reported an increase in weight during the lockdown; a recent Italian survey reported a weight gain in 46.6\% of the sample. ${ }^{14}$ Comparably, the present study's results showed that the mean and SD of their weight during lockdown was higher than at their last clinic visit prelockdown (Tables 1 \& 2). In addition, we calculated the patients' BMI to detect if there was any difference before and during the lockdown. A significant increase in BMI and body weight during lockdown was found (Table 2).

Impaired glycemic control was exacerbated by decreased physical activity and an unhealthy diet. The results of this study found that $52.2 \%$ of participants followed a healthy diet before lockdown, compared to $23.2 \%$ during lockdown (Table 3). This result was similar to that of a European study, which reported an increase in appetite by $34.4 \%$ of the respondents. ${ }^{14} \mathrm{~A}$ number of online surveys around the world agreed that food consumption and dietary patterns were unhealthier during confinement. ${ }^{15}$

In this study, it was found that $46.4 \%$ had difficulty in following a healthy diet during the lockdown, owing to increased consumption of fast food, carbohydrates, and soft drinks (Table 3).

It has long been recognized that a sedentary lifestyle has a detrimental impact on glycemic control, and conversely, that physical activity has a significant role in the regulation of blood glucose. ${ }^{16}$ Lockdown policies in the current pandemic have caused massive distortion in people's daily routines, including their physical activity level. Physical activity decreased in $66.1 \%$ of our patients during the lockdown compared to before lockdown. This was a function of sedentary habits during their leisure time, namely, watching TV, playing online games, and using computers. Hence, glycemic control was adversely affected during the lockdown.

Good glycemic control can be achieved via frequent home monitoring of blood glucose levels and following up with regular $\mathrm{HbA1c}$ tests. Diabetic patients tended to face difficulties attending their pre-scheduled follow-up visits, and, as a consequence, many patients postponed or even canceled such visits. In this study, we analyzed the frequency of daily home glucose measurements and found that the majority of our patients were using a continuous glucose sensor (Figure 1). By comparing their blood glucose readings before and during the lockdown, a significant $(p=0.007)$ increase in glucose readings was demonstrated. 
Patients with T1D depend on insulin therapy for glycemic control. ${ }^{17}$ The present study found that $27.1 \%$ had difficulty in obtaining insulin during the lockdown, which may have reflected on their readings.

Diabetic patients are more likely to suffer from stress and mood deterioration, which could be exacerbated by circumstances such as a lockdown. According to our findings, $59.6 \%$ of the subjects suffered from mood deterioration, possibly affecting their blood glucose readings.

Study limitations. There are 5 major limitations in this study, which could be addressed in future research. First, for technical reasons, we were unable to obtain data about the average number of glucose readings per patient that were used to calculate mean glucose in patients who were not on CGMS (continuous glucose monitoring system) Second, we could not separate the analysis on participants who were on continuous glucose monitoring to confirm our findings. Third, this study involved a small number of patients; therefore, our findings may not be generalizable to the entire population of Saudi Arabia. The fourth limitation was the use of convenience sampling, which may aggravate the risk of selection bias. Future studies should involve a larger sample size. The fifth limitation was that the compliance of the participants with their regimen was not assessed.

In conclusion, many patients reported a change in their lifestyle and eating habits during the lockdown, which likely predisposed them to difficulties in glycemic control.

Acknowledgment. This research article was made possible with the help and support of Nada O. Alrowashed and Jamla A. Msfer Alshahrani. We would like to thank Scribendi company (Scribendi.com) for English language editing.

\section{References}

1. Bell GI, Polonsky KS. Diabetes mellitus and genetically programmed defects in $\beta$-cell function. Nature [Internet] 2001; 414: 788-791.

2. Katsarou A, Gudbjörnsdottir S, Rawshani A, Dabelea D, Bonifacio E, Anderson BJ, et al. Type 1 diabetes mellitus. Nat Rev Dis Prim 2017; 3: 17016.

3. Bonora BM, Boscari F, Avogaro A, Bruttomesso D, Fadini GP. Glycaemic control among people with type 1 diabetes during lockdown for the SARS-CoV-2 outbreak in Italy. Diabetes Ther 2020; 11: 1369-1379.
4. Khandelwal S. Psychosocial illness in children with type 1 diabetes mellitus: prevalence, pattern and risk factors. J Clin Diagnostic Res 2016; 10: SC05-SC08.

5. Gupta R, Ghosh A, Singh AK, Misra A. Clinical considerations for patients with diabetes in times of COVID-19 epidemic. Diabetes Metab Syndr Clin Res Rev 2020; 14: 211-212.

6. Verma A, Rajput R, Verma S, Balania VKB, Jangra B. Impact of lockdown in COVID 19 on glycemic control in patients with type 1 Diabetes Mellitus. Diabetes Metab Syndr Clin Res Rev 2020; 14: 1213-1216.

7. Nachimuthu S, Vijayalakshmi R, Sudha M, Viswanathan V. Coping with diabetes during the COVID - 19 lockdown in India: Results of an online pilot survey. Diabetes Metab Syndr Clin Res Rev 2020; 14: 579-582.

8. Hussain A, Bhowmik B, do Vale Moreira NC. COVID-19 and diabetes: Knowledge in progress. Diabetes Res Clin Pract 2020; 162: 108142.

9. Fernández E, Cortazar A, Bellido V. Impact of COVID-19 lockdown on glycemic control in patients with type 1 diabetes. Diabetes Res Clin Pract 2020; 166: 108348.

10. Yıldız E. What can be said about lifestyle and psychosocial issues during the coronavirus disease pandemic? First impressions. Perspect Psychiatr Care 2020; 6: 10.1111/ppc. 12560.

11. World Health Organization. New WHO-led study says majority of adolescents worldwide are not sufficiently physically active [Internet]. [cited 2020]. Available from: https://www. who.int/news-room/detail/22-11-2019-new-who-led-studysays- majority-of-adolescents-worldwide-are-not-sufficientlyphysically-active-putting-their-current-and-future-health-atrisk

12. Ammar A, Brach M, Trabelsi K, Chtourou H, Boukhris O, Masmoudi L, et al. Effects of COVID-19 home confinement on eating behaviour and physical activity: Results of the ECLBCOVID19 International Online Survey. Nutrients 2020; 12: 1583.

13. Xiang $M$, Zhang Z, Kuwahara K. Impact of COVID-19 pandemic on children and adolescents' lifestyle behavior larger than expected. Prog Cardiovasc Dis 2020; 63: 531-532.

14. Di Renzo L, Gualtieri P, Pivari F, Soldati L, Attinà A, Cinelli $\mathrm{G}$, et al. Eating habits and lifestyle changes during COVID-19 lockdown: an Italian survey. J Transl Med 2020; 18: 229.

15. Deschasaux-Tanguy M, Druesne-Pecollo N, Esseddik Y, Szabo de Edelenyi F, Alles B, Andreeva VA, et al. Diet and physical activity during the COVID-19 lockdown period (March-May 2020): results from the French NutriNet-Sante cohort study. [cited 2020]. Available from: http://medrxiv.org/content/early /2020/06/05/2020.06.04.20121855.abstract

16. Guo L, Ren L, Yang S, Xiao M, Chang D, Yang F, et al. Profiling early humoral response to diagnose novel coronavirus disease (COVID-19). Clin Infect Dis 2020; 71: 778-785.

17. DiMeglio LA, Evans-Molina C, Oram RA. Type 1 diabetes. Lancet 2018; 391: 2449-2462. 